OPEN ACCESS

Edited by:

Alessio Molfino,

Sapienza University of Rome, Italy

Reviewed by:

Lina Ma,

Capital Medical University, China

Caterina Conte,

Università telematica San

Raffaele, Italy

${ }^{*}$ Correspondence:

Xinjuan Wu

wuxinjuan@sina.com

Tao Xu

xutaosd@126.com

tThese authors share first authorship

Specialty section:

This article was submitted to

Clinical Nutrition,

a section of the journal

Frontiers in Nutrition

Received: 12 November 2020 Accepted: 03 June 2021

Published: 29 June 2021

Citation:

Zhang X-M, Jiao J, Zhu C, Guo N, Liu Y, Lv D, Wang H, Jin J, Wen $X$,

Zhao S, Wu X and Xu T (2021) Association Between Low Handgrip Strength and 90-Day Mortality Among

Older Chinese Inpatients: A National Multicenter Prospective Cohort Study.

Front. Nutr. 8:628628.

doi: 10.3389/fnut.2021.628628

\section{Association Between Low Handgrip Strength and 90-Day Mortality Among Older Chinese Inpatients: A National Multicenter Prospective Cohort Study}

\begin{abstract}
Xiao-Ming Zhang ${ }^{1 \dagger}$, Jing Jiao ${ }^{1 \dagger}$, Chen Zhu ${ }^{1}, N_{a}$ Guo ${ }^{1}$, Ying Liu ${ }^{1}$, Dongmei Lv $^{2}$, Hui Wang ${ }^{3}$, Jingfen $\mathrm{Jin}^{4}$, Xianxiu Wen ${ }^{5}$, Shengxiu Zhao ${ }^{6}$, Xinjuan $\mathrm{Wu}^{1 *}$ and Tao $\mathrm{Xu}^{7 *}$

${ }^{1}$ Department of Nursing, Chinese Academy of Medical Sciences - Peking Union Medical College, Peking Union Medical College Hospital, Beijing, China, ${ }^{2}$ Department of Nursing, The Second Affiliated Hospital of Harbin Medical University, Harbin, China, ${ }^{3}$ Department of Nursing, Tongji Hospital, Tongji Medical College, Huazhong University of Science and Technology, Wuhan, China, ${ }^{4}$ Department of Nursing, The Second Affiliated Hospital, Zhejiang University School of Medicine, Hangzhou, China, ${ }^{5}$ Department of Nursing, Sichuan Provincial People's Hospital, Chengdu, China, ${ }^{6}$ Department of Nursing, Qinghai Provincial People's Hospital, Xining, China, ${ }^{7}$ Department of Epidemiology and Statistics, Institute of Basic Medical Sciences, Chinese Academy of Medical Sciences and School of Basic Medicine, Peking Union Medical College, Beijing, China
\end{abstract}

Background: The knowledge of the association between low handgrip strength and mortality among older Chinese inpatients is limited. Given China's aging society, a great number of older adults require hospital admission.

Objective: To explore the association between low handgrip strength and 90-day mortality, providing evidence for clinicians to predict the risk of mortality and improve clinical outcomes for older inpatients.

Materials and Methods: We conducted a national multicenter cohort study with a baseline survey from October 2018 to February 2019 and followed up for 90 days to record mortality outcomes. The assessment of handgrip strength was conducted using a hand dynamometer with the cutoff (handgrip strength $<28 \mathrm{~kg}$ for men and $<18 \mathrm{~kg}$ for women) to define low handgrip strength. Multivariable logistic regression analysis was applied to explore the association between low handgrip strength and 90-day mortality.

Results: A total of 8,910 older Chinese inpatients [mean (SD) age, 72.39 (5.68) years; 3,750 women (42.09\%)], with a prevalence of low handgrip strength, at $49.57 \%$, were included. Compared to inpatients with normal handgrip strength, inpatients with low handgrip strength were older, had less education, more were female, had lower activities of daily living (ADL) score, had lower BMI, higher frailty, higher rates of depression, and poorer cognitive function (all $p<0.05$ ). At 90 days, after adjusting for gender, age, education, frailty, depression, $A D L$ score, malnutrition, and diagnosis, low handgrip strength was independently associated with 90-day mortality, compared to normal handgrip strength $(\mathrm{OR}=1.64,95 \% \mathrm{Cl}: 1.14-2.37 ; P=0.008)$. Additionally, subgroup and interaction analysis showed a significant interaction effect $(P=0.031)$ between two age groups (65-74 years older vs. $\geq 75$ years old), with the OR being 3.19 (95\%Cl:2.07-4.93) and 1.49 (95\%Cl:0.87-2.55), respectively. 
Conclusion: Older Chinese inpatients with low handgrip strength had a 1.64fold risk of 90-day mortality, compared to those with normal handgrip strength, indicating that clinicians need to screen early for handgrip strength and recommend corresponding interventions, such as resistance training and nutrition, as a priority for older inpatients.

Clinical Trial Registration: Chinese Clinical Trial Registry, Identifier: ChiCTR1800017682.

Keywords: handgrip strength, older adults, mortality, inpatient, cohort study

\section{INTRODUCTION}

The number of older adults is experiencing remarkable growth worldwide, meaning that the world's population is aging, especially in China. It is estimated there will be $\sim 350$ million older adults worldwide by 2050 (https://population.un.org/wpp/ DataQuery). Thus, healthcare for older adults is a challenge for every country. One of the main characteristics of aging is a significant change in body composition in terms of decreased lean body mass and incremental increased fat mass (1). Older adults often experience declining skeletal muscle, which makes up the major part of lean body mass, along with existing poor muscle strength. Muscle strength is a core component in maintaining physical ability, which assists older adults in sustaining better functional status (2). Older adults often suffer from low muscle strength, mainly due to the aging mechanism, unhealthy behaviors or lifestyle, or health status/comorbidities $(3,4)$.

Low muscle strength has been confirmed to be strongly associated with adverse outcomes. Handgrip strength is considered a better parameter to reflect whole-body strength because it provides reliable, simple, and rapid standardized measurements. According to the European Working Group on Sarcopenia in Older People (EWGSOP), low handgrip strength is an important component in defining sarcopenia (5). It is widely reported that low handgrip strength is associated with a decline in cognitive function (6), low quality of life (7), incidence of disability, and even mortality (8).

Recently, numerous studies have reported that patients with low handgrip strength are at increased risk of mortality or cardiovascular disease (CVD) mortality in different settings, such as in the community and hospital, in various countries (911). However, few studies have been conducted in a Chinese population. Chua et al. (12) conducted a cohort study with 13,789 community-dwelling adults, indicating that handgrip strength was inversely associated with mortality risk, the figure of HR being 2.05 (95\%CI:1.44-2.90) compared to the extreme quartiles. However, this Chinese population was from Singapore, possessing different characteristics from Mainland China residents, as Singaporeans enjoy a high level of healthcare and standard of living. Also, the Chua et al. study (12) did not perform subgroup or interaction analysis based on gender,

Abbreviations: EWGSOP, European Working Group on Sarcopenia in Older People; HR, hazard ratio; OR, odds ratio; SD, Standard deviation; BMI, Body mass index; CI, Confidence interval. age, frailty, or depression. Meanwhile, Zhuang et al. (13), who conducted a large-scale study of 8,267 cancer patients in China, found that low handgrip is strongly associated with an increased risk of cancer mortality. As mentioned above, these two studies explored this association for a Chinese population; however, studies examining the association between low handgrip strength and 90-day mortality in older Chinese hospitalized patients are limited. Therefore, our study aimed to investigate the association between low handgrip strength (HS $<28 \mathrm{~kg}$ for men and $<18 \mathrm{~kg}$ for women) based on The Asian Working Group for Sarcopenia (AWGS) (14) and 90-day mortality in a large-scale prospective cohort study at multiple centers in China. In addition, we have employed these associations after adjusting different covariates, and conducted a subgroup analysis to determine whether the impact of low handgrip can be different in various groups.

\section{MATERIALS AND METHODS \\ Study Design and Participants}

This was a large-scale prospective cohort study, which originally explored the prevalence of frailty and associated factors, at multiple centers in China (15). Our project was approved by the ethics committee of Peking Union Medical College Hospital. In addition, we have registered our protocols in the Chinese Clinical Trial Registry (ChiCTR1800017682) (15). In general, there are three levels of hospital in China: primary hospitals, secondary hospitals, and tertiary hospitals. Primary and secondary hospitals are primary health care institutions and regional hospitals, respectively. In comparison, tertiary hospitals are large urban referral and comprehensive hospitals, with a bed capacity exceeding 500 . Tertiary hospitals are responsible for providing comprehensive medical, education, and scientific research, and can serve as medical centers providing care to multiple regions. Our study was focused on older hospitalized patients in tertiary hospitals. According to the original study design (15), we used a formula to calculate sample size. Based on the previous study, the prevalence of frailty was about $25 \%$, and a $95 \%$ confidence level with a margin of error of $10 \%$ was defined. Therefore, considering possible non-response, 1,4001,800 inpatients were recruited for each hospital, resulting in a total of 10,000 inpatients. A baseline survey was conducted from October 2018 to February 2019.

\section{Sampling Methods and Study Population}

The cluster sampling methods consisted of three stages. First, we chose six provinces or municipalities according to 
China's administrative regions (Southwest, Northeast, North, Northwest, Southcentral, and East) with simple random sampling, eventually choosing Sichuan Province, Heilongjiang Province, Hubei Province, Beijing municipality, Qinghai Province, and Zhejiang Province. The researcher then selected each tertiary hospital from the aforementioned regions by using the same simple random sampling. Finally, patients from several departments, including surgery, medicine, neurology, orthopedics, and ICU, who met the inclusion and exclusion criteria, were continuously recruited from these five hospitals. Our inclusion criteria are defined as follows: age $\geq 65$ years older; volunteered to participate in this project, and signed the consent form. Older hospitalized patients were excluded if they or their caregivers could not communicate effectively with the investigators.

\section{Handgrip Strength Measurement}

Trained investigators employ a standard procedure to measure handgrip strength. First, an older patient stands and keeps their upper body upright, holding the handgrip dynamometer naturally down, and then pressing with maximum force for $2 \mathrm{~s}$, with the data recorded in this moment. Handgrip strength was measured twice with each hand. Final handgrip strength was recorded based on maximal hand strength. Low handgrip strength was defined according to the Asian Working Group for Sarcopenia (AWGS), with a figure of $\mathrm{HS}<28 \mathrm{~kg}$ for men and $<18 \mathrm{~kg}$ for women (14).

\section{Covariates Definition}

Inpatient demographic variables, including gender, age, education, marital status, and ethnicity were collected within $48 \mathrm{~h}$ after hospital admission. Meanwhile, we also investigated other important covariates-such as smoking status, alcohol use, mobility (being bedridden), vision, hearing, sleep, urinary function, primary diagnosis, and length of hospitalization-of which we defined the variables of vision, hearing, sleep, and urinary function as dysfunction when they affected normal life. Geriatric syndromes-such as depression, frailty, cognitive impairment, and nutritional status-were also assessed. The FRAIL scale was used, with five simple questions to assess five domains (fatigue, ambulation, loss of weight, resistance, and illness). Frailty was considered with a score $\geq 3$ (16). We also used the Geriatric Depression Scale 15 (GDS15) score instrument to evaluate whether inpatients had depression (17). According to this instrument, when the total score is five or more, patients can be defined as having depression. For cognitive impairment, we used a Chinese version of Mini-Cog, consisting of two components: a clock drawing test and a three-item recall task (defined by scores $\leq 2$ ) (18). Mini-Nutritional Assessment-Short Form was applied to classify the nutrition category (defined normal nutritional status 12-14; scoring from 8 to 11 points was classified as being at risk of malnutrition, while being malnourished was defined as scoring 0-7 points) (19). We also collected the Activities of Daily Living using the Barthel Index.

\section{Mortality Assessment}

Trained investigators performed a telephone follow-up for mortality 90 days after the baseline survey. The investigators were systematically trained for this procedure to make the outcomes reliable and credible.

\section{Statistical Analysis}

The difference between two groups, such as low handgrip strength vs. normal handgrip strength, and deceased vs. survivors, was detected by Student's $t$-test and chi-squared test or Fisher's Exact Test when the data were displayed by continuous variables (mean \pm standard deviation) and categorical data (percentage), respectively. There were $\sim 200$ primary diagnoses among these patients because of the participants who were drawn from different departments, including medicine, surgery, and other departments. For data analysis, we classified these patients into five categories (heart disease, pulmonary diseases, other internal medicine diseases, surgical diseases, and cancer). A generalized additive model (GAM) and smooth curve fitting analysis were used to identify whether there was a non-linear correlation between handgrip strength and 90-day mortality. Univariate analysis and multivariable logistic regression models were also adopted to detect the association between low handgrip strength and 90-day mortality. We listed an unadjusted model and different adjusted models according to the demographic characteristics or geriatric syndromes. Finally, subgroup and interaction were adopted to identify whether the association between low handgrip strength and 90-day mortality changed in different groups. A two-sided at alpha $=0.05$ was adopted for all statistical analysis and performed using SAS9.4 software (SAS Institute Inc., Cary, NC, USA).

\section{RESULTS}

\section{Demographics}

A total of 9,996 patients was recruited by our team at the initial stage, with 9,301 patients remaining after checking and managing the raw data due to 695 participants failing to finish the handgrip strength assessment. In addition, we eventually confirmed 8,910 patients for analysis because 391 patients could not be contacted at 90 days for the mortality assessment (Figure 1). Overall, the average age of this cohort was $72.39(\mathrm{SD}=5.68)$ years, including $3,750(42.09 \%)$ women. The distribution of education categories was $15.91 \%$ for illiterate, $28.66 \%$ for primary school, $40.72 \%$ for middle school, and $14.72 \%$ for university. Other detailed information is shown in Table $\mathbf{1}$.

\section{Comparison Between Low Handgrip Strength and Normal Handgrip Strength}

Table 1 describes general characteristics and other variables between low handgrip strength and normal handgrip strength. Overall, the prevalence of low handgrip strength was $49.57 \%$. Hospitalized patients with low handgrip strength were older, had less education, more were female, and they scored lower in terms of ADL and BMI. A higher percentage of patients with low handgrip strength had been bedridden for an extended period and suffered from vision and sleep dysfunction. In addition, 


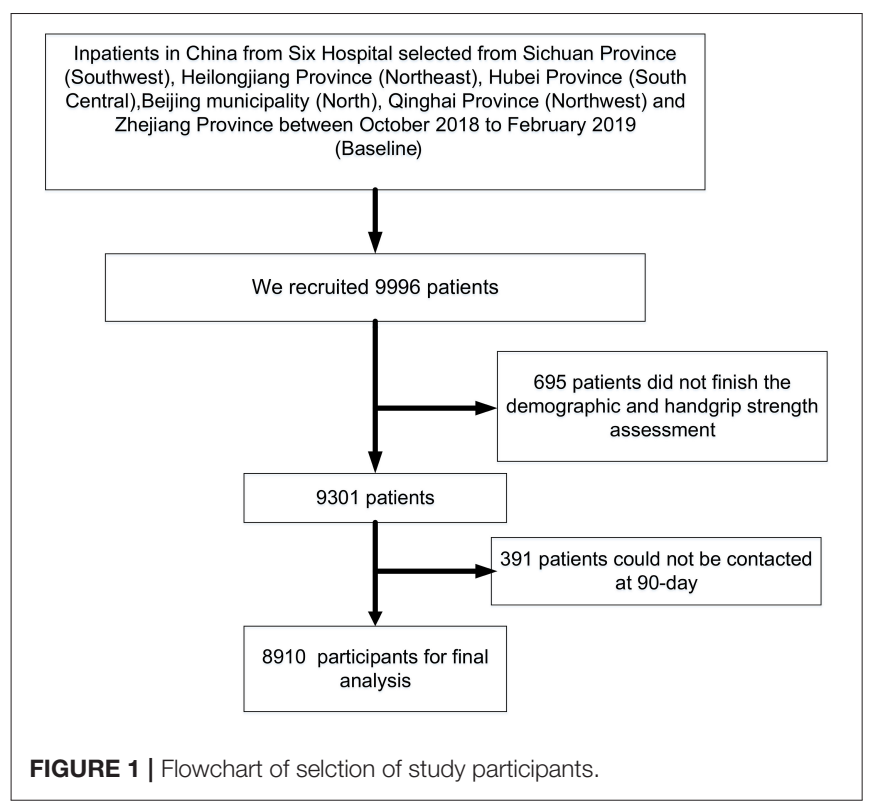

low handgrip strength was associated with cognitive impairment (all $P<0.01)$, malnourishment $(P<0.01)$, frailty $(P<0.01)$, and depression $(P<0.01)$. The proportion of low handgrip strength in cancer patients was higher than in patients with heart disease and pulmonary disease (15.88 vs. 11.69 vs. $7.50 \%)$. The difference in length of hospital stay was statistically significant between those with low handgrip strength and those with normal handgrip strength $(P<0.01)$.

\section{Univariate Analysis}

There was a statistically significant difference between survivors and those who were deceased at 90 days in terms of age, ADL, BMI, gender, smoking status, alcohol use, being bedridden for an extended period, depression, Mini Nutritional Assessment short-form (MNA-SF), frailty, and length of hospital stay, with all $P$-value being $<0.05$. Additionally, the rate of 90 -day mortality was higher in the low handgrip strength group compared to the normal handgrip strength group, with a significant difference (2.7 vs. $1.0 \%, P<0.0001$ ). However, we did not find any significant differences between these groups in terms of ethnicity, education, marital status, vision, hearing, and sleep. The highest rate of 90-day mortality (4.34\%) among all disease groups was in cancer patients. Meanwhile, the rate of 90-day mortality between patients with cognitive impairment and those with normal cognitive function was very close $(2.4$ vs. $1.7 \%, P=$ 0.0492). All results are shown in Table 2.

\section{Non-linear Relationship Analyses}

A non-linear relationship between handgrip strength and 90day mortality was performed, with the results presenting a negative linear relationship. This result indicates that with an increase in handgrip strength, the 90-day mortality rate decreased (Figure 2).
TABLE 1 | Prevalence conditions of low handgrip strength across demographics.

\begin{tabular}{|c|c|c|c|c|}
\hline Variables & Overall & $\begin{array}{l}\text { Low handgrip } \\
\text { strength }\end{array}$ & $\begin{array}{c}\text { Normal } \\
\text { handgrip } \\
\text { strength }\end{array}$ & $P$-value \\
\hline Sample size & 8,910 & $4,417(49.57)$ & 4,493 (50.43) & \\
\hline Age (years) (mean, SD) & $72.39 \pm 5.68$ & $73.58 \pm 6.07$ & $71.22 \pm 4.98$ & $<0.0001$ \\
\hline ADL (mean, SD) & $28.00 \pm 4.03$ & $26.98 \pm 4.83$ & $28.99 \pm 2.68$ & $<0.0001$ \\
\hline BMI (kg/m²) (mean, SD) & $23.61 \pm 3.49$ & $23.06 \pm 3.58$ & $24.15 \pm 3.31$ & $<0.0001$ \\
\hline Gender ( $n, \%)$ & & & & 0.018 \\
\hline Female & 3,750 (42.09) & 1,914 (51.04) & 1,836 (48.96) & \\
\hline Male & $5,160(57.91)$ & 2,503 (48.51) & 2,657 (51.49) & \\
\hline Ethnicity (n, \%) & & & & $<0.0001$ \\
\hline Han & 8,376 (94.01) & 4,095 (48.89) & 4,281 (51.11) & \\
\hline Other & 534 (5.99) & 322 (60.30) & $212(39.70)$ & \\
\hline Education ( $n, \%)$ & & & & $<0.001$ \\
\hline Illiterate & $1,417(15.91)$ & $904(63.80)$ & $513(36.20)$ & \\
\hline Primary school & 2,553 (28.66) & 1,404 (54.99) & $1,149(45.01)$ & \\
\hline Middle school & 3,628 (40.72) & $1,600(44.10)$ & $2,028(55.90)$ & \\
\hline University & $1,311(14.72)$ & 509 (38.83) & $802(61.17)$ & \\
\hline Marital status $(n, \%)$ & & & & $<0.001$ \\
\hline Marriage & 7,902 (88.80) & $3,826(48.42)$ & 4,076 (51.58) & \\
\hline Divorced or widowed & $997(11.20)$ & $584(58.58)$ & $413(41.42)$ & \\
\hline $\begin{array}{l}\text { Primary diagnosis }(n \text {, } \\
\%)\end{array}$ & & & & $<0.001$ \\
\hline Heart disease & $1,181(13.26)$ & 516 (11.69) & $665(14.80)$ & \\
\hline Pulmonary disease & $6,13(6.88)$ & $331(7.50)$ & $282(6.28)$ & \\
\hline $\begin{array}{l}\text { Other internal medicine } \\
\text { disease }\end{array}$ & 2,713 (30.46) & $1,520(34.43)$ & 1,193 (26.55) & \\
\hline Surgical disease & 2,925 (32.84) & 1,347 (30.51) & $1,578(17.25)$ & \\
\hline Cancer & $1,476(16.57)$ & $701(15.88)$ & 775 (35.12) & \\
\hline Smoking status $(n, \%)$ & & & & $<0.001$ \\
\hline Non-smoker & $5,880(65.99)$ & 2,978 (50.65) & 2,902 (49.35) & \\
\hline Current smoker & $991(11.12)$ & 427 (43.09) & $564(56.91)$ & \\
\hline Former smoker & 2,039 (22.88) & 1,012 (49.63) & 1,027 (50.37) & \\
\hline $\begin{array}{l}\text { Alcohol consumption } \\
(n, \%)\end{array}$ & & & & $<0.001$ \\
\hline Non-drinker & 6,805 (76.37) & $3,438(50.52)$ & 3,367 (49.48) & \\
\hline Current drinker & $1,036(11.63)$ & $429(41.41)$ & 607 (58.59) & \\
\hline Former drinker & $1,069(12.00)$ & $550(51.45)$ & $519(48.55)$ & \\
\hline Bedridden $(n, \%)$ & & & & $<0.001$ \\
\hline Yes & 205 (2.3) & $156(76.10)$ & 49 (23.90) & \\
\hline No & 8,705 (97.70) & 4,261 (48.95) & 4,444 (51.05) & \\
\hline Vision (n, \%) & & & & $<0.001$ \\
\hline Dysfunction & $1,943(21.81)$ & 1,053 (54.19) & $890(45.81)$ & \\
\hline Normal & 6,967 (78.19) & 3,364 (48.28) & 3,603 (51.72) & \\
\hline Hearing (n, \%) & & & & $<0.001$ \\
\hline Dysfunction & 1,674 (18.79) & 948 (56.63) & $726(43.37)$ & \\
\hline Normal & $7,236(81.21)$ & 3,469 (47.94) & 3,767 (52.06) & \\
\hline Sleep $(n, \%)$ & & & & $<0.001$ \\
\hline Dysfunction & $3,802(42.67)$ & $2,049(53.89)$ & $1,753(46.11)$ & \\
\hline Normal & 5,108 (57.33) & 2,368 (46.36) & $2,740(53.64)$ & \\
\hline Urinary function $(n, \%)$ & & & & $<0.001$ \\
\hline Dysfunction & $1,197(13.43)$ & $682(56.98)$ & 515 (43.02) & \\
\hline Normal & $7,713(86.57)$ & 3,735 (48.42) & $3,978(51.58)$ & \\
\hline
\end{tabular}


TABLE 1 | Continued

\begin{tabular}{lcccc}
\hline Variables & Overall & $\begin{array}{c}\text { Low handgrip } \\
\text { strength }\end{array}$ & $\begin{array}{c}\text { Normal } \\
\text { handgrip } \\
\text { strength }\end{array}$ & P-value \\
\hline $\begin{array}{l}\text { Depression (n, \%) } \\
\text { Yes }\end{array}$ & $1,369(15.64)$ & $920(67.20)$ & $449(32.80)$ & $<0.001$ \\
No & $7,385(84.36)$ & $3,410(46.17)$ & $3,975(53.83)$ & \\
Cognitive impairment & & & & $<0.001$ \\
(n, \%) & $1,684(19.77)$ & $1,098(65.20)$ & $586(34.80)$ & \\
Yes & $6,832(80.23)$ & $3,069(44.92)$ & $3,763(55.08)$ & \\
No & & & & $<0.001$ \\
MNA-SF (n, \%) & $4,999(56.11)$ & $2,030(40.61)$ & $2,969(59.39)$ & \\
$\begin{array}{l}\text { Normal nutritional } \\
\text { status }\end{array}$ & $3,044(34.16)$ & $1,761(57.85)$ & $1,283(42.15)$ & \\
At risk of malnutrition & $867(9.73)$ & $626(72.20)$ & $241(27.80)$ & \\
Malnourished & & & & $<0.001$ \\
Frailty ( $n$, \%) & $1,492(16.75)$ & $1,075(72.05)$ & $417(27.95)$ & \\
Yes & $7,418(83.25)$ & $3,342(45.05)$ & $4,076(54.95)$ & \\
No & $9.15 \pm 5.84$ & $9.61 \pm 5.94$ & $8.70 \pm 5.72$ & $<0.001$ \\
Length of hospital stay & & & & \\
(days) (mean, SD) & & & & \\
\hline
\end{tabular}

$A D L$, basic activities of daily living; MNA-SF, Mini-Nutritional Assessment-Short Form.

\section{The Relationship Between Low Handgrip Strength and 90-day Mortality}

The multivariable logistic regression analysis indicated that low handgrip strength was associated with an increased risk of 90day mortality in an unadjusted model (OR $=2.57,95 \% \mathrm{CI}: 1.83-$ 3.61; $P<0.001)$. This association was diminished when adjusting different variables. After fully adjusting for age, gender, education, frailty, depression, malnutrition, ADL, and primary diagnosis, this association remained (OR $=1.64,95 \% \mathrm{CI}: 1.14-$ 2.37; $P=0.008$ ). Detailed information is presented in Table 3. Furthermore, we also did a subgroup based on stratified and interaction analysis and found that the OR value of low handgrip strength and 90-day mortality was stronger in patients aged 6574 years old than in the group aged 75 years old or more (OR $=3.19 ; 95 \%$ CI:2.07-4.93, vs. OR $=1.49,95 \%$ CI:0.87-2.55), with a significant interaction effect $(P=0.031)$. In the other group, we did not find any significant interaction in terms of gender, depression, frailty, primary diagnosis, and MNA-SF (All $P$-value $>0.05$ ) (Figure 3).

\section{DISCUSSION}

Our study indicates that older Chinese inpatients with low handgrip strength are at increased risk of 90-day mortality, compared to those with normal handgrip strength, after adjusting relevant demographic characteristics and other covariates, such as depression, frailty, ADL, primary diagnosis, and MNA-SF. It indicates that low handgrip strength could be a prognostic factor for clinicians to stratify groups at high risk of mortality. Additionally, subgroup and interaction analysis showed that this association was higher in the 65- to 74-year-old age group than
TABLE 2 | Univariate analysis results.

\begin{tabular}{|c|c|c|c|}
\hline Variables & $\begin{array}{c}\text { Survivors at } \\
90 \text {-day }\end{array}$ & $\begin{array}{l}\text { Deceased at } \\
\text { 90-day }\end{array}$ & $P$-value \\
\hline Sample size & 8,743 & 167 & \\
\hline Age (years) (mean, SD) & $72.36 \pm 5.66$ & $73.70 \pm 6.19$ & 0.002 \\
\hline ADL (mean, SD) & $28.03 \pm 4.00$ & $26.40 \pm 4.85$ & $<0.0001$ \\
\hline $\begin{array}{l}\text { Low handgrip strength }(n \text {, } \\
\%)\end{array}$ & & & $<0.0001$ \\
\hline Yes & 4,298 (97.31) & $119(2.69)$ & \\
\hline NO & 4445 (98.93) & $48(1.07)$ & \\
\hline BMI $\left(\mathrm{kg} / \mathrm{m}^{2}\right)$ (mean, SD) & $23.65 \pm 3.47$ & $21.82 \pm 3.72$ & $<.0001$ \\
\hline Gender (n, \%) & & & 0.004 \\
\hline Female & 3,702 (98.72) & $48(1.28)$ & \\
\hline Male & $5,041(97.69)$ & $119(2.31)$ & \\
\hline Ethnicity $(n, \%)$ & & & 0.9977 \\
\hline Han & $8,219(98.13)$ & $157(1.87)$ & \\
\hline Other & $524(98.13)$ & $10(1.87)$ & \\
\hline Education (n, \%) & & & 0.1309 \\
\hline Illiterate & $1,386(97.81)$ & $31(2.19)$ & \\
\hline Primary & 2,512 (98.39) & $41(1.61)$ & \\
\hline Middle & $3,550(97.85)$ & $78(2.15)$ & \\
\hline University & $1,294(98.70)$ & $17(1.30)$ & \\
\hline Marital status (n, \%) & & & 0.2534 \\
\hline Marriage & $7,750(98.08)$ & $152(1.92)$ & \\
\hline Divorced or widowed & $983(98.60)$ & $14(1.40)$ & \\
\hline Primary diagnosis (n, \%) & & & $<0.001$ \\
\hline Heart disease & $1,170(99.07)$ & $11(0.93)$ & \\
\hline Pulmonary disease & $594(96.90)$ & $19(3.10)$ & \\
\hline $\begin{array}{l}\text { Other internal } \\
\text { medicine diseases }\end{array}$ & $2,670(98.42)$ & $43(1.58)$ & \\
\hline Surgical diseases & 2,895 (98.97) & $30(1.03)$ & \\
\hline Cancer & $1,412(95.66)$ & $64(4.34)$ & \\
\hline Smoking status ( $n, \%)$ & & & 0.021 \\
\hline Non-smoker & $5,775(98.21)$ & $105(1.79)$ & \\
\hline Current smoker & $980(98.89)$ & $11(1.11)$ & \\
\hline Former smoker & $1,988(97.50)$ & $51(2.50)$ & \\
\hline Alcohol consumption ( $n, \%)$ & & & 0.034 \\
\hline Non-drinker & 6,682 (98.19) & $123(1.81)$ & \\
\hline Current drinker & 1,022 (98.65) & $14(1.35)$ & \\
\hline Former drinker & $1,039(97.19)$ & $30(2.81)$ & \\
\hline Bedridden (n, \%) & & & 0.0013 \\
\hline Yes & 195 (95.12) & $10(4.88)$ & \\
\hline No & $8,548(98.20)$ & $157(1.80)$ & \\
\hline Vision (n, \%) & & & 0.4033 \\
\hline Dysfunction & $1,911(98.35)$ & $32(1.65)$ & \\
\hline Normal & 6,832 (98.06) & $135(1.94)$ & \\
\hline Hearing $(n, \%)$ & & & 0.7453 \\
\hline Dysfunction & $1,641(98.03)$ & $33(1.97)$ & \\
\hline Normal & $7,102(98.15)$ & $134(1.85)$ & \\
\hline Sleep $(n, \%)$ & & & 0.2216 \\
\hline Dysfunction & 3,723 (97.92) & $79(2.08)$ & \\
\hline Normal & $5,020(98.28)$ & $88(1.72)$ & \\
\hline Urinary function $(n, \%)$ & & & 0.049 \\
\hline Dysfunction & $1,166(97.41)$ & $31(2.59)$ & \\
\hline
\end{tabular}

(Continued) 
TABLE 2 | Continued

\begin{tabular}{lccc}
\hline Variables & $\begin{array}{c}\text { Survivors at } \\
\text { 90-day }\end{array}$ & $\begin{array}{c}\text { Deceased at } \\
\text { 90-day }\end{array}$ & P-value \\
\hline Normal & $7,577(98.24)$ & $136(1.76)$ & \\
Depression $(n, \%)$ & $1,322(96.57)$ & $47(3.43)$ & $<0.001$ \\
Yes & $7,265(98.38)$ & $120(1.62)$ & \\
No & & & 0.0492 \\
Cognitive impairment $(n, \%)$ & $1,643(97.57)$ & $41(2.43)$ & \\
Yes & $6,715(98.29)$ & $117(1.71)$ & \\
No & & & $<0.001$ \\
MNA-SF (n, \%) & $4,962(99.26)$ & $37(0.74)$ & \\
Normal nutritional status & $2,967(97.47)$ & $77(2.53)$ & \\
At risk of malnutrition & $814(93.89)$ & $53(6.11)$ & \\
Malnourished & & & $<0.001$ \\
Frailty ( $n$, \%) & $1,428(95.71)$ & $64(4.29)$ & \\
Yes & $7,315(98.61)$ & $103(1.39)$ & \\
No & $9.11 \pm 5.80$ & $11.20 \pm 7.72$ & $<0.001$ \\
Length of hospital stay & & & \\
(days) (mean, SD) & & & \\
\hline
\end{tabular}

ADL, basic activities of daily living;MNA-SF, Mini-Nutritional Assessment-Short Form.

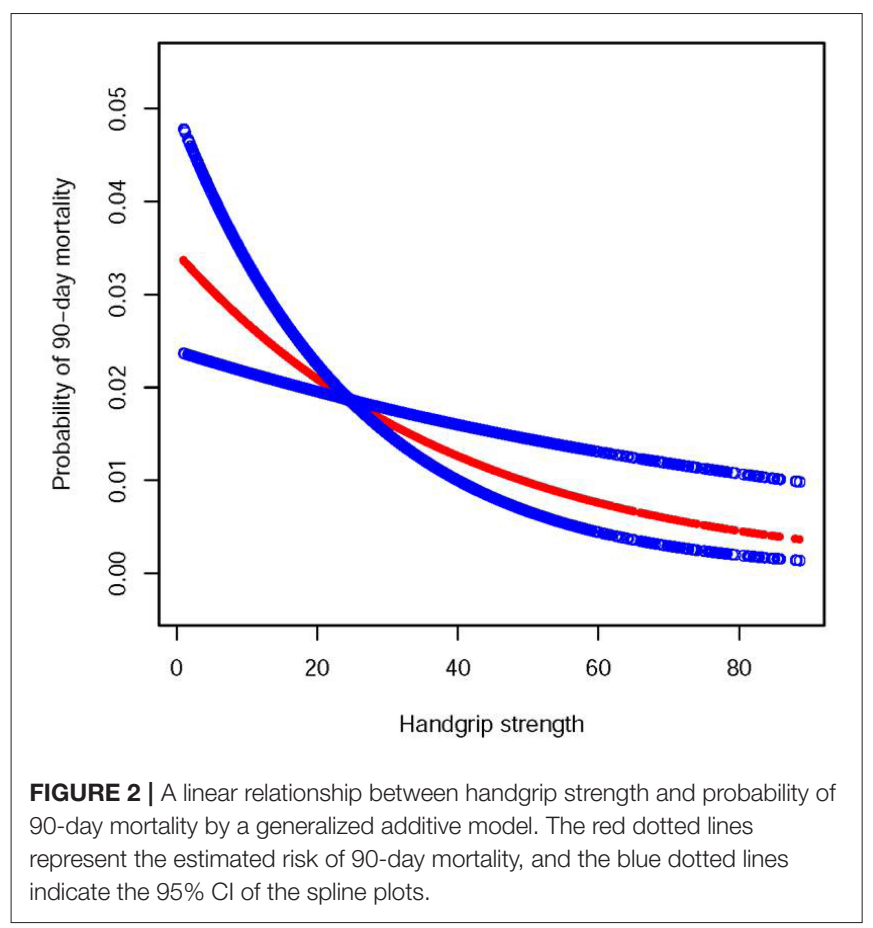

in the group aged 75 years or older. This is one of the few studies, to the best of our knowledge, exploring the association between low handgrip strength and 90-day mortality in older Chinese inpatients covering various diseases.

Overall, there is a great number of studies reporting that community-dwelling older adults with low handgrip strength are at increased risk of mortality or CVD mortality. In a systematic review and meta-analysis published in 2018 (20), including 38 studies with a total of $1,907,580$ participants, the findings revealed that higher handgrip strength levels were associated with a reduced risk of mortality (pooled $\mathrm{HR}=0.69$; 95\% CI, 0.64-0.74). In comparison, this meta-analysis only included one study on older Chinese adults, with only 99 participants. A recent larger study on the Chinese population conducted by Zhuang et al. (13), exploring the correlation between handgrip strength (HGS) and mortality, showed that older adults with low HGS had a strongly increased risk of overall cancer mortality. In contrast, participants in our study were general inpatients and suffered from various types of disease, not exclusively from cancer. In our study, the prevalence of low handgrip strength was $49.57 \%$, higher than in the study by Zhuang et al. (13) (25.7\%). The difference may be due to the fact that the average age in our study was much higher, at 72.39 vs. 58 years old. It is reported that handgrip strength gradually declines with age (21). Thus, our study also indicates that low handgrip strength is prevalent among older Chinese inpatients.

Interestingly, we found the association between low handgrip strength and 90-day mortality was stronger in patients ages 6574 years old than in those aged $\geq 75$ years, with a significant interaction difference $(P=0.031)$. Consistent with our study, Celis-Morales et al. (22) reported that HR of the association between lower handgrip strength and all-cause mortality was higher in the younger age group than in the older age group. In addition, other studies described similar results among cancer patients (13). As we all know, the older the person, the lower the handgrip strength (23). In our study, average handgrip strength was higher in the younger age group than in the older age group $(25.68 \pm 12.00 \mathrm{~kg}$ vs. $22.01 \pm 10.83 \mathrm{~kg} ; p<0.001)$. Why did we not observe that the impact of handgrip strength has a worse risk of mortality in those aged $\geq 75$ years? We speculate that older inpatients have worse health, often suffering from multiple comorbidities, such as diabetes, hypertension, frailty, and cognitive impairment. Thus, these conditions conceal the effects of low handgrip strength on mortality. More studies about the age effect on low handgrip strength and mortality in older inpatients are needed to explore this question.

We found the impact of handgrip strength on 90-day mortality was similar between genders. However, in terms of the gender-specific effects of handgrip on mortality, we were unable to draw a conclusion based on the literature. Arvandi et al. (24), reported that hazard ratio $(95 \% \mathrm{CI})$ of handgrip strength on mortality was higher in women than in men. Three studies found that low handgrip strength was not associated with mortality in women (25-27). The mechanism causing this discrepancy was unclear. Different ethnicities, hormones, study design, and sample size may have led to this inconsistent result. Thus, more large well-designed prospective cohort studies are warranted in future to examine this important issue.

Older inpatients often suffer from depression after enduring disease for an extended period. It is estimated that the prevalence of depression among inpatients ranges from 25.1 to $57.5 \%$ (28). Low handgrip strength coexisting with depression among older patients is very common (29). Our subgroup analysis results showed that the OR of mortality was higher in the group with depression than in the group without depression. However, we need to be careful that the interaction analysis of depression was 
TABLE 3 | Multivariable logistic regression analysis of the association between low handgrip strength and 90-day mortality.

\begin{tabular}{|c|c|c|c|c|c|}
\hline Exposure & $\begin{array}{l}\text { Non-adjusted } \\
(\mathrm{OR}, 95 \%, \mathrm{Cl})\end{array}$ & $\begin{array}{c}\text { Adjusted I } \\
\text { (OR, 95\%, Cl) }\end{array}$ & $\begin{array}{c}\text { Adjusted II } \\
\text { (OR, 95\%, Cl,) }\end{array}$ & $\begin{array}{c}\text { Adjusted III } \\
(\mathrm{OR}, 95 \%, \mathrm{Cl})\end{array}$ & $\begin{array}{l}\text { Adjusted IV } \\
(\mathrm{OR}, 95 \%, \mathrm{Cl})\end{array}$ \\
\hline \multicolumn{6}{|c|}{ LOWER HANDGRIP STRENGTH } \\
\hline No & Reference & Reference & Reference & Reference & Reference \\
\hline Yes & $\begin{array}{c}2.57 \\
(1.83-3.61) ;<0.001\end{array}$ & $\begin{array}{c}2.43 \\
(1.71-3.45) ;<0.001\end{array}$ & $\begin{array}{c}2.04 \\
(1.43-2.92) ;<0.001\end{array}$ & $\begin{array}{c}1.97 \\
(1.38-2.84) ; 0.0002\end{array}$ & $\begin{array}{c}1.64 \\
(1.14,2.37) 0.008\end{array}$ \\
\hline
\end{tabular}

Adjusted l: age gender education.

Adjusted II: age gender education, frailty.

Adjusted III: age gender education, frailty, depression.

Adjusted IV: age gender education, frailty, depression, ADL. Primary diagnosis.

Subgroup analysis
Age
$65-74$ years
>=75 years
Gender
Male
Female
Primary diagnosis
Heart diseases
Pulmonary diseases
Other Internal diseases
Surgical Diseases
Cancer diseases
Depression
Yes
No
Frailty
Yes
No
Nutritional status
Normal
At risk of malnutrition
Malnourished

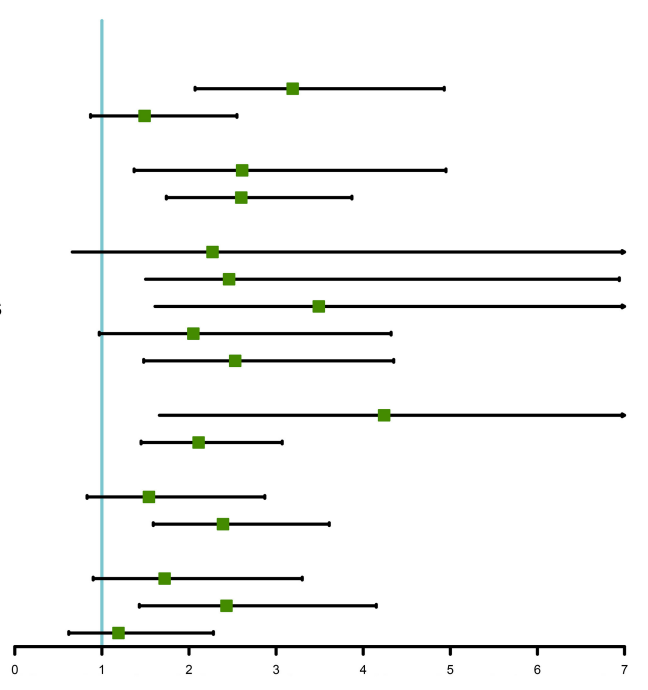

Odds Ration OR(95\%Cl)

$3.19(2.07,4.93)$
$1.49(0.87,2.55)$

1.49(0.87,2.55)

$2.61(1.37,4.95)$

$2.60(1.74,3.87)$

2.27(0.66,7.80)

$2.46(1.87,6.94)$

$3.49(1.61,7.56)$

2.05(0.97,4.32)

2.53(1.48,4.35)

$4.24(1.66,10.81)$

$2.11(1.45,3.07)$

1.54(0.83,2.87)

$2.39(1.59,3.61)$

1.72(0.90,3.30)

$2.43(1.43,4.15)$

$1.19(0.62,2.28)$

\section{$P$ for interaction \\ 0.031 \\ 0.982}

0.351

0.174

0.234

1.135

FIGURE 3 | Subgroup analysis of the association between low handgrip strength and 90-day mortality.

not significant. One study reported by Park et al. (30) indicated that older adults with low handgrip strength coexisting with depression are at higher risk of mortality, compared to those experiencing low handgrip strength alone. The main reason may be that people suffering from depression have been reported to be at increased risk of mortality (31) and depression, which are related to low handgrip strength (32), with both factors combined leading to increased mortality risk.

The underlying mechanisms behind the association between low handgrip strength and mortality are not clearly understood, but appear to be complicated and multifactorial. First, low handgrip strength and lower muscle mass are the two main components that define sarcopenia, a condition confirmed by numerous studies to lead to a greater risk of mortality (33-36). In addition, low handgrip strength to some extent reflects decreased skeletal muscle mass due to aging, because sufficient skeletal muscle mass plays a crucial role in maintaining strength, and skeletal muscle mass is the main organ for metabolism. Aging and malnutrition lead to decreased muscle mass, a slowing metabolic rate, and reduced calorie consumption (37). This impact is intensified by inactivity, in turn giving rise to a high risk of metabolic syndrome (obesity, diabetes, dyslipidemia), eventually increasing the likelihood of mortality (38). Third, low handgrip strength can partially be explained by diminished neural system functioning, which results in poor physical function and can lead to a high risk of falls (39). As we all know, falls are one of the main reasons for a greater risk of mortality among inpatients (40). Therefore, this complicated association and mechanism warrants further exploration.

\section{CLINICAL IMPLICATIONS}

Our study indicated that low handgrip strength could be an independent predictor for mortality among Chinese inpatients, which is consistent with other previously published studies $(12,41)$. Handgrip strength can be obtained by using simple equipment that is not time-consuming to use and can be applied in any clinical setting, which is very convenient for medical staff (42). In addition, according to the EWGSOP2 recommendation, muscle strength is a core component of screening for sarcopenia, because it can reflect key features of the disease, and has been reported to accompany an increased risk of adverse outcomes (5). Thus, screening inpatients for muscle strength can have multiple clinical implications. First, clinicians can stratify 
high-risk inpatients and initiate an intervention programsuch as physical therapy, an exercise training program, and comprehensive treatment-early on, in order to reduce mortality rates. Second, clinicians can educate patients' family members to pay attention to their loved ones, for example, by suggesting a home-based resistance and aerobic exercise program to improve muscle strength.

Our study has both strengths and limitations. To the best of our knowledge, this is one of the few large sample studies of Chinese inpatients covering various types of disease based on a multiple-center prospect cohort study to explore the association between handgrip strength and 90-day mortality. In addition, we performed comprehensive analyses, such as generalized additive model (GAM), multivariable logistic regressions for adjusting variables, such as geriatric syndrome (frailty, depression, malnutrition, ADL) not controlled in previous studies, and subgroup and interaction analysis. Most importantly, our study could encourage medical staff to consider screening for handgrip strength as an important routine measurement in older hospitalized patients. At the same time, with these limitations, there must also be cautions. First, we recruited participants from different regions of China, which means that we need to be cautious about generalizing our findings to other countries. Second, we did not collect the parameter that represents muscle mass; thus, we cannot calculate sarcopenia and investigate the association between sarcopenia and 90-day mortality. Third, the follow-up length of our study was relatively short-term, which prevents us from comparing our findings to other studies that included long-term outcomes $(10,11)$. Fourth, although our study was a prospective cohort study, we cannot draw a definite conclusion on causal inference. Fifth, we subjectively classified these diagnoses into five groups, which might produce some bias. Sixth, the collection of data from six different tertiary hospitals; although the level of the medical service at tertiary hospitals is regulated in a standardized way in mainland China, we still cannot totally exclude the possible effect of the different hospitals themselves on our results.

\section{CONCLUSION}

We reported that low handgrip strength is strongly associated with 90-day mortality among older Chinese hospitalized patients,

\section{REFERENCES}

1. Fielding RA, Vellas B, Evans WJ, Bhasin S, Morley JE, Newman AB, et al. Sarcopenia: an undiagnosed condition in older adults. Current consensus definition: prevalence, etiology, and consequences. International working group on sarcopenia. J Am Med Dir Assoc. (2011) 12:24956. doi: 10.1016/j.jamda.2011.01.003

2. Reid KF, Fielding RA. Skeletal muscle power: a critical determinant of physical functioning in older adults. Exerc Sport Sci Rev. (2012) 40:412. doi: 10.1097/JES.0b013e31823b5f13

3. Rom O, Karkabi K, Reznick AZ, Keidar Z, Aizenbud D. Relationship between history of smoking, metabolic and inflammatory markers, parameters of body composition and muscle strength. Adv Exp Med Biol. (2015) 849:4956. doi: 10.1007/5584_2014_92

4. Yorke AM, Curtis AB, Shoemaker M, Vangsnes E. Grip strength values stratified by age, gender, and chronic disease status in even adjusting for demographic characteristics, depression, frailty, malnutrition, ADL, and diagnosis, indicating the usefulness of handgrip strength applied in a clinical setting for assessing and improving prognosis. Early screening and comprehensive interventions, such as nutrition and exercise programs, might be important for older hospitalized patients.

\section{DATA AVAILABILITY STATEMENT}

The raw data supporting the conclusions of this article will be made available by the authors, without undue reservation.

\section{ETHICS STATEMENT}

The studies involving human participants were reviewed and approved by Ethics Committee of Peking Union Medical College Hospital (S-K540). Written informed consent forms to participate were obtained in the study. The patients/participants provided their written informed consent to participate in this study.

\section{AUTHOR CONTRIBUTIONS}

$\mathrm{XW}$ is responsible for designing this study and initiating this concept. X-MZ and JJiao were responsible for drafting the initial manuscript. TX conducted all statistical analyses. CZ, NG, YL, DL, HW, JJin, XW, and SZ were responsible for collecting the raw data and organizing this project. All authors contributed to the article and approved the submitted version.

\section{FUNDING}

This study was funded by Peking Union Medical College with grant number 2018PT33001.

\section{ACKNOWLEDGMENTS}

We sincerely thank all of the inpatients for their participation and understanding. In addition, all of the researchers and managers in charge of this project provided us with a great deal of support. We also thank everyone who participated in our program.

adults aged 50 years and older. J Geriatr Phys Ther. (2015) 38:115-21. doi: 10.1519/JPT.0000000000000037

5. Cruz-Jentoft AJ, Bahat G, Bauer J, Boirie Y, Bruyère O, Cederholm $\mathrm{T}$, et al. Sarcopenia: revised European consensus on definition and diagnosis. Age Ageing. (2019) 48:16-31. doi: 10.1093/ageing/ afy169

6. Sui SX, Holloway-Kew KL, Hyde NK, Williams LJ, Leach S, Pasco JA. Muscle strength and gait speed rather than lean mass are better indicators for poor cognitive function in older men. Sci Rep. (2020) 10:10367. doi: 10.1038/s41598-020-67251-8

7. Chan W, Chin SH, Whittaker AC, Jones D, Kaur O, Bosch JA. The associations of muscle strength, muscle mass, and adiposity with clinical outcomes and quality of life in prevalent kidney transplant recipients. J Ren Nutr. (2019) 29:536-47. doi: 10.1053/j.jrn.2019.06.009

8. Soysal P, Hurst C, Demurtas J, Firth J, Howden R, Yang L, et al. Handgrip strength and health outcomes: umbrella review of systematic reviews with 
meta-analyses of observational studies. J Sport Health Sci. (2020) 10:2905. doi: 10.1016/j.jshs.2020.06.009

9. Laukkanen JA, Voutilainen A, Kurl S, Araujo CGS, Jae SY, Kunutsor SK. Handgrip strength is inversely associated with fatal cardiovascular and all-cause mortality events. Ann Med. (2020) 52:109-19. doi: 10.1080/07853890.2020.1748220

10. Bae EJ, Park NJ, Sohn HS, Kim YH. Handgrip strength and all-cause mortality in middle-aged and older Koreans. Int J Environ Res Public Health. (2019) 16:740. doi: 10.3390/ijerph16050740

11. Pérez-Rodríguez P, Rabes-Rodríguez L, Sáez-Nieto C, Alarcón TA, Queipo R, Otero-Puime Á, et al. Handgrip strength predicts 1-year functional recovery and mortality in hip fracture patients. Maturitas. (2020) 141:205. doi: 10.1016/j.maturitas.2020.06.013

12. Chua KY, Lim WS, Lin X, Yuan JM, Koh WP. Handgrip strength and timed up-and-go (TUG) test are predictors of short-term mortality among elderly in a population-based cohort in Singapore. J Nutr Health Aging. (2020) 24:371-8. doi: 10.1007/s12603-020-1337-0

13. Zhuang CL, Zhang FM, Li W, Wang $\mathrm{KH}, \mathrm{Xu} \mathrm{HX}$, Song $\mathrm{CH}$, et al. Associations of low handgrip strength with cancer mortality: a multicentre observational study. J Cachexia Sarcopenia Muscle. (2020) 11:147686. doi: $10.1002 / \mathrm{jcsm} .12614$

14. Chen LK, Liu LK, Woo J, Assantachai P, Auyeung TW, Bahyah KS, et al. Sarcopenia in Asia: consensus report of the Asian Working Group for Sarcopenia. J Am Med Dir Assoc. (2014) 15:95-101. doi: 10.1016/j.jamda.2013.11.025

15. Jiao J, Wang Y, Zhu C, Li F, Zhu M, Wen X, et al. Prevalence and associated factors for frailty among elder patients in China: a multicentre cross-sectional study. BMC Geriatr. (2020) 20:100. doi: 10.1186/s12877-020-1496-1

16. Kojima G. Frailty defined by FRAIL scale as a predictor of mortality: a systematic review and meta-analysis. J Am Med Dir Assoc. (2018) 19:4803. doi: 10.1016/j.jamda.2018.04.006

17. Guerin JM, Copersino ML, Schretlen DJ. Clinical utility of the 15-item geriatric depression scale (GDS-15) for use with young and middleaged adults. J Affect Disord. (2018) 241:59-62. doi: 10.1016/j.jad.201 8.07.038

18. Borson S, Scanlan JM, Watanabe J, Tu SP, Lessig M. Simplifying detection of cognitive impairment: comparison of the Mini-Cog and Mini-Mental State Examination in a multiethnic sample. J Am Geriatr Soc. (2005) 53:8714. doi: 10.1111/j.1532-5415.2005.53269.x

19. Soysal P, Veronese N, Arik F, Kalan U, Smith L, Isik AT. Mini Nutritional Assessment Scale-Short Form can be useful for frailty screening in older adults. Clin Interv Aging. (2019) 14:693-9. doi: 10.2147/CIA.S196770

20. García-Hermoso A, Cavero-Redondo I, Ramírez-Vélez R, Ruiz JR, Ortega FB, Lee DC, et al. Muscular strength as a predictor of all-cause mortality in an apparently healthy population: a systematic review and meta-analysis of data from approximately 2 million men and women. Arch Phys Med Rehabil. (2018) 99:2100-13.e2105. doi: 10.1016/j.apmr.2018.01.008

21. Wearing J, Konings P, Stokes M, de Bruin ED. Handgrip strength in old and oldest old Swiss adults - a cross-sectional study. BMC Geriatr. (2018) 18:266. doi: 10.1186/s12877-018-0959-0

22. Celis-Morales CA, Welsh P, Lyall DM, Steell L, Petermann F, Anderson J, et al. Associations of grip strength with cardiovascular, respiratory, and cancer outcomes and all cause mortality: prospective cohort study of half a million UK Biobank participants. BMJ. (2018) 361:k1651. doi: 10.1136/bmj.k1651

23. Taniguchi Y, Fujiwara Y, Murayama H, Yokota I, Matsuo E, Seino S, et al. Prospective Study of trajectories of physical performance and mortality among community-dwelling older Japanese. J Gerontol A Biol Sci Med Sci. (2016) 71:1492-9. doi: 10.1093/gerona/glw029

24. Arvandi M, Strasser B, Meisinger C, Volaklis K, Gothe RM, Siebert U, et al. Gender differences in the association between grip strength and mortality in older adults: results from the KORA-age study. BMC Geriatr. (2016) 16:201. doi: 10.1186/s12877-016-0381-4

25. Gale CR, Martyn CN, Cooper C, Sayer AA. Grip strength, body composition, and mortality. Int J Epidemiol. (2007) 36:228-35. doi: 10.1093/ije/dyl224

26. Fujita Y, Nakamura Y, Hiraoka J, Kobayashi K, Sakata K, Nagai $\mathrm{M}$, et al. Physical-strength tests and mortality among visitors to health-promotion centers in Japan. J Clin Epidemiol. (1995) 48:1349-59. doi: 10.1016/0895-4356(95)00533-1

27. Katzmarzyk PT, Craig CL. Musculoskeletal fitness and risk of mortality. Med SciSports Exerc. (2002) 34:740-4. doi: 10.1097/00005768-200205000-00002
28. Xu T, Jiao J, Zhu C, Li F, Guo X, Li J, et al. prevalence and potential associated factors of depression among chinese older inpatients. J Nutr Health Aging. (2019) 23:997-1003. doi: 10.1007/s12603-019-1270-2

29. Marconcin P, Peralta M, Ferrari G, Gaspar de Matos M, Espanha M, Murawska-Ciałowicz E, et al. The association of grip strength with depressive symptoms among middle-aged and older adults with different chronic diseases. Int J Environ Res Public Health. (2020) 17:6942. doi: 10.3390/ijerph17196942

30. Park S, Cho J, Kim D, Jin Y, Lee I, Hong H, et al. Handgrip strength, depression, and all-cause mortality in Korean older adults. BMC Geriatr. (2019) 19:127. doi: 10.1186/s12877-019-1140-0

31. Almeida OP, Hankey GJ, Yeap BB, Golledge J, Norman PE, Flicker L. Depression, frailty, and all-cause mortality: a cohort study of men older than 75 years. J Am Med Dir Assoc. (2015) 16:296-300. doi: 10.1016/j.jamda.2014.10.023

32. Marques A, Gomez-Baya D, Peralta M, Frasquilho D, Santos T, Martins J. The effect of muscular strength on depression symptoms in adults: a systematic review and meta-analysis. Int J Environ Res Public Health. (2020) 17:5674. doi: 10.3390/ijerph17165674

33. Chen LK, Woo J, Assantachai P, Auyeung TW, Chou MY, Iijima K, et al. Asian working group for sarcopenia: 2019 consensus update on sarcopenia diagnosis and treatment. J Am Med Dir Assoc. (2020) 21:3007.e302. doi: 10.1016/j.jamda.2019.12.012

34. Xia L, Zhao R, Wan Q, Wu Y, Zhou Y, Wang Y, et al. Sarcopenia and adverse health-related outcomes: An umbrella review of meta-analyses of observational studies. Cancer Med. (2020) 9:7964-78. doi: 10.1002/cam4.3428

35. Ubachs J, Ziemons J, Minis-Rutten IJG, Kruitwagen R, Kleijnen J, Lambrechts S, et al. Sarcopenia and ovarian cancer survival: a systematic review and meta-analysis. J Cachexia Sarcopenia Muscle. (2019) 10:116574. doi: $10.1002 /$ jcsm. 12468

36. Zhang X, Wang C, Dou Q, Zhang W, Yang Y, Xie X. Sarcopenia as a predictor of all-cause mortality among older nursing home residents: a systematic review and meta-analysis. BMJ Open. (2018) 8:e021252. doi: 10.1136/bmjopen-2017-021252

37. Argilés JM, Campos N, Lopez-Pedrosa JM, Rueda R, RodriguezMañas L. Skeletal muscle regulates metabolism via interorgan crosstalk: roles in health and disease. J Am Med Dir Assoc. (2016) 17:789-96. doi: 10.1016/j.jamda.2016.04.019

38. Kalyani RR, Corriere M, Ferrucci L. Age-related and disease-related muscle loss: the effect of diabetes, obesity, and other diseases. Lancet Diabetes Endocrinol. (2014) 2:819-29. doi: 10.1016/S2213-8587(14)7 0034-8

39. McGrath R, Vincent BM, Peterson MD, Jurivich DA, Dahl LJ, Hackney $\mathrm{KJ}$, et al. Weakness may have a causal association with early mortality in older Americans: a matched cohort analysis. J Am Med Dir Assoc. (2020) 21:621-6.e622. doi: 10.1016/j.jamda.2019.10.016

40. Burns E, Kakara R. Deaths from falls among persons aged $\geq 65$ Years United States, 2007-2016. MMWR Morb Mortal Wkly Rep. (2018) 67:50914. doi: $10.15585 / \mathrm{mmwr} . \mathrm{mm} 6718 \mathrm{al}$

41. Chen PJ, Lin MH, Peng LN, Liu CL, Chang CW, Lin YT, et al. Predicting cause-specific mortality of older men living in the Veterans home by handgrip strength and walking speed: a 3-year, prospective cohort study in Taiwan. J Am Med Dir Assoc. (2012) 13:517-21. doi: 10.1016/j.jamda.201 2.02 .002

42. Aliberti MJR, Szlejf C, Covinsky KE, Lee SJ, Jacob-Filho W, Suemoto CK. Prognostic value of a rapid sarcopenia measure in acutely ill older adults. Clin Nutr. (2020) 39:2114-20. doi: 10.1016/j.clnu.201 9.08.026

Conflict of Interest: The authors declare that the research was conducted in the absence of any commercial or financial relationships that could be construed as a potential conflict of interest.

Copyright $\odot 2021$ Zhang, Jiao, Zhu, Guo, Liu, Lv, Wang, Jin, Wen, Zhao, Wu and Xu. This is an open-access article distributed under the terms of the Creative Commons Attribution License (CC BY). The use, distribution or reproduction in other forums is permitted, provided the original author(s) and the copyright owner(s) are credited and that the original publication in this journal is cited, in accordance with accepted academic practice. No use, distribution or reproduction is permitted which does not comply with these terms. 